\title{
Diffusion-weighted imaging of injuries to the visual centers of the brain in patients with type 2 diabetes and retinopathy
}

\author{
MING LIANG ${ }^{1}$, XING CHEN $^{1}$, FENG XUE ${ }^{2}$, LINGNA MENG $^{3}$ and YUNFENG CONG ${ }^{1}$ \\ ${ }^{1}$ Urgent Care Centre; Departments of ${ }^{2}$ Internal Medical Oncology and ${ }^{3}$ Stomatology, Nangang Branch, \\ Heilongjiang Provincial Hospital, Harbin, Heilongjiang 150001, P.R. China
}

Received February 26, 2016; Accepted March 10, 2017

DOI: $10.3892 /$ etm.2017.4582

\begin{abstract}
The present study aimed to investigate the ability of diffusion-weighted imaging (DWI) to identify injury to the visual centers of the brain in patients with type 2 diabetes with retinopathy. The study included 84 cases ( 63 patients with type 2 diabetic retinopathy and 21 healthy individuals) that were assessed using DWI. Diabetic patients were equally divided into three groups: Proliferative diabetic retinopathy (PDR), non-proliferative diabetic retinopathy (NPDR) and diabetic without retinopathy. The results demonstrated that individuals in the PDR group had significantly higher disease duration and glycated hemoglobin levels than the diabetic without retinopathy group $(\mathrm{P}<0.05)$. Apparent diffusion coefficient (ADC) values were significantly higher in functional brain areas of the PDR group compared with the NPDR group $(\mathrm{P}<0.001)$, whose values were significantly higher compared with the diabetic without retinopathy and control groups $(\mathrm{P}<0.001)$. In addition, glycated hemoglobin levels and disease duration were positively correlated with mean ADC values in the same functional areas of the brain. In conclusion, DWI-measured ADC values may be an effective indicator of brain dysfunction in individuals with type 2 diabetic retinopathy. DWI is able to assess brain injury in individuals with early diabetic retinopathy, which may make the diagnostic technique a useful predictor of early ocular disease.
\end{abstract}

\section{Introduction}

Diabetic retinopathy (DR), or damage to blood vessels of the retina, is a serious complication of diabetes. With the rising incidence of diabetes, DR is a common ocular fundus lesion and a leading cause of blindness and visual impairment (1-4).

Correspondence to: Dr Yunfeng Cong, Urgent Care Centre, Nangang Branch, Heilongjiang Provincial Hospital, 82 Zhongshan Road, Harbin, Heilongjiang 150001, P.R. China

E-mail: congyunfeng24@163.com

Key words: diabetic retinopathy, diffusion-weighted imaging, visual center, glycated hemoglobin, apparent diffusion coefficient
Worldwide prevalence of DR is 30-60\% of individuals with diabetes and the prevalence in China is 35.6-63.5\% (1-4).

Diffusion-weighted imaging (DWI) is a functional magnetic resonance imaging (MRI) technique that may be used in the central nervous system and is able to effectively provide information on pathological changes in the brain (5). DWI has become an important approach for radiographic diagnosis of brain lesions, including Parkinson's disease, tumors and cerebral apoplexy, as well as liver diseases (6-8). DWI may be used to calculate the apparent diffusion coefficient (ADC), a measure of brain injury, as it assesses diffusion of water molecules from blood vessels (9).

Early identification of DR is important to manage the disease and prevent progression to blindness. DR has been associated with injury to visual centers of the brain using DWI-measured ADC values (10). As the pathogenesis of brain injury is not fully understood, an effective approach to detect early injuries is required to improve the prognosis of individuals with DR.

The present study explored the correlation between DR and functional brain injury by comparing clinical data and weighted imaging from 63 individuals with type 2 diabetes and 21 healthy control individuals.

\section{Subjects and methods}

Subjects. The present study was approved by the Ethics Committee of Heilongjiang Provincial Hospital (Harbin, China) and informed consent was obtained from all subjects. The study cohort included 63 individuals with type 2 diabetes who were admitted to the Heilongjiang Provincial Hospital between April 2014 and April 2015. Of the 63 diabetic individuals, 31 were male $(49.21 \%)$ and 32 were female (50.79\%). Type 2 diabetes was diagnosed using the criteria established by the American Diabetes Association $(11,12)$.

Based on funduscopy and fundus fluorescein angiography, diabetic individuals were divided into three groups. Group 1 included 21 proliferative diabetic retinopathy (PDR) cases, of which 11 were male (52.38\%) and 10 were female (47.62\%). Group 1 had a mean age of $54.95 \pm 10.86$ years, a mean disease duration of $11.92 \pm 6.59$ years and symptoms including vitreous hemorrhage and preretinal hemorrhage. Group 2 was composed of 21 non-proliferative diabetic retinopathy (NPDR) cases, of which 10 were male $(47.62 \%)$ and 11 were 
female (52.38\%). Group 2 had a mean age of $55.10 \pm 8.95$ years, a mean disease duration of $8.12 \pm 3.71$ years and symptoms including retinal hemorrhage and microangiomas. Group 3 included 21 diabetic without retinopathy cases, of which 10 were male (47.62\%) and 11 were female (52.38\%). Group 3 had a mean age of $54.73 \pm 6.05$ years and a mean disease duration of $5.67 \pm 2.48$ years.

The study also included 21 healthy volunteers who received examinations at Heilongjiang Provincial Hospital during the same period. Of the 21 healthy individuals, 11 were male $(52.38 \%)$ and 10 were female $(47.62 \%)$, with a mean age of $55.12 \pm 7.60$ years. The diagnostic criteria for healthy volunteers were as follows: No type 2 diabetes; no cataracts, glaucoma or other eye lesions; no history of symptomatic cerebral apoplexy; and no other brain diseases. Regarding sex and age, there were no significant differences between the PDR, NPDR, diabetic without retinopathy and healthy control groups $(\mathrm{P}>0.05)$.

Data collection. Patients' blood glucose and glycated hemoglobin (HbAlc) levels were measured $10 \mathrm{~h}$ after fasting. Following this, fundus fluorescein angiography was performed. The immunoturbidimetry reagents for detection of HbAlc, matched quality control and calibration were manufactured by Randox Laboratories, Ltd. (Crumlin, UK). The tests were conducted on an automatic biochemistry analyzer (Hitachi 7600; Hitachi, Ltd., Tokyo, Japan) for quality control using fresh blood with anticoagulant ethylenediaminetetraacetic acid-K2 (Humica Weihai International Co., Ltd., Weihai, China).

MRI. A Philips Intera Master 3.0T superconducting MR scanner (Philips Medical Systems, Eindhoven, The Netherlands) was used for all MRI. Scan sequences included DWI, fluid-attenuated inversion recovery (FLAIR), T1-weighted imaging (T1WI) and T2-weighted imaging (T2WI). Scan parameters were as follows: DWI, echo time (TE) $=60 \mathrm{msec}$ and repetition time (TR)=1,924 msec; FLAIR, TE=136 msec and TR=8,700 msec; T1WI, TE=15 msec and $\mathrm{TR}=560 \mathrm{msec}$; and T2WI, TE=61 $\mathrm{msec}$ and TR=2,363 msec. Other parameters included: Field of view of $230 \mathrm{~mm}$; matrix size of $128 \times 128 \mathrm{~mm}$; number of excitations of 2; slice thickness of $4 \mathrm{~mm}$; number of slices of 25 ; scan time of $28 \mathrm{sec}$; slice gap of $1 \mathrm{~mm}$; and diffusion sensitivity of 0 or $1,000 \mathrm{sec} / \mathrm{mm}^{2}$.

$A D C$. ADC was used as an index of the magnitude of diffusion, and mean ADC values were calculated to analyze diffusion changes. As previously described, seven regions of interest (ROI) in the brain were selected, and their ADC values were measured (10). When selecting ROI, regions that contained cerebrospinal fluid and artifacts were avoided to preserve the accuracy of ADC values. The areas of measured regions included: Thalamus, 50-60 $\mathrm{mm}^{2}$; visual cortex, $80-100 \mathrm{~mm}^{2}$; corona radiate, $70-80 \mathrm{~mm}^{2}$; dorsolateral frontal cortex; cingulate gyrus; dorsomedial frontal cortex; and orbitofrontal cortex, 30-40 $\mathrm{mm}^{2}$ (Fig. 1).

Statistical analysis. Double data entry was performed using EpiData version 3.1 software (EpiData Association, Odense, Denmark) to create a data bank, and logic checks were performed with SAS version 9.2 software (SAS Institute, Inc.,
Table I. Demographic and clinical features of patients.

\begin{tabular}{|c|c|c|c|}
\hline Group & $\mathrm{n}$ & $\begin{array}{c}\text { Disease } \\
\text { duration, years }\end{array}$ & HbAlc, $\%$ \\
\hline PDR & 21 & $12.25 \pm 7.03^{\mathrm{a}}$ & $9.71 \pm 2.73$ \\
\hline NPDR & 21 & $8.24 \pm 3.59$ & $7.92 \pm 1.68$ \\
\hline $\begin{array}{l}\text { Diabetic without } \\
\text { retinopathy }\end{array}$ & 21 & $5.57 \pm 2.73$ & $7.13 \pm 0.84$ \\
\hline$F$ & & 8.47 & 6.95 \\
\hline P-value & & $<0.001$ & 0.004 \\
\hline
\end{tabular}

${ }^{a} \mathrm{P}<0.05$ vs. the diabetic without retinopathy group. Data are presented as the mean \pm standard deviation. HbAlc, glycosylated hemoglobin; PDR, proliferative diabetic retinopathy; NPDR, non-proliferative diabetic retinopathy.
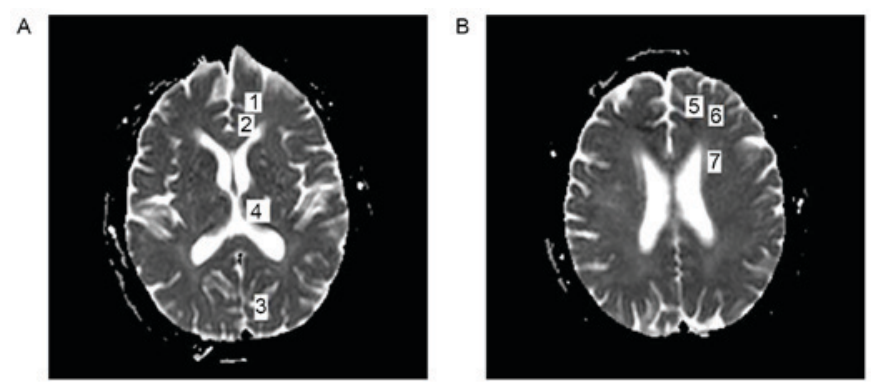

Figure 1. Measured areas of functional brain ROI. (A) ROI 1-4, including orbitofrontal cortex, cingulate gyrus, visual cortex and thalamus, respectively. (B) ROI 5-7, including dorsomedial frontal cortex, dorsolateral frontal cortex and corona radiate, respectively. ROI, regions of interest.

Cary, NC, USA). Statistical methods included analysis of variance (ANOVA) with Student-Newman-Keuls (SNK) method for comparison among multiple means and Spearman's rho correlation. MedCalc (version 16.2; MedCalc Software, Ostend, Belgium) was used to draw receiver operating characteristic (ROC) curves. Data are presented as the mean \pm standard deviation. $\mathrm{P}<0.05$ was considered to indicate a statistically significant difference.

\section{Results}

Comparison of disease duration and HbAlc levels. ANOVA demonstrated that disease duration $(\mathrm{P}<0.001)$ and HbAlc levels $(P=0.004)$ were significantly different among the PDR, NPDR and diabetic without retinopathy groups (Table I). However, SNK method comparison demonstrated that disease duration was only significantly different between the PDR and diabetic without retinopathy groups, with PDR having a longer disease duration than the diabetic without retinopathy group $(\mathrm{P}<0.05)$. HbAlc levels were also significantly higher in the PDR group than in the diabetic without retinopathy group $(\mathrm{P}<0.05)$; however, this did not differ significantly between the other groups.

Comparison of mean ADC values in functional areas of the brain. Mean ADC values in cingulate gyri, orbitofrontal 
Table III. Correlation of ADC with disease duration and $\mathrm{HbAlc}$ level.

\begin{tabular}{lccc}
\hline & \multicolumn{3}{c}{ ADC, R (P) } \\
\cline { 2 - 4 } Variable & Cingulate gyrus & OFC & Visual cortex \\
\hline HbAlc & $0.287(0.047)$ & $0.328(0.021)$ & $0.361(0.015)$ \\
$\begin{array}{l}\text { Disease } \\
\text { duration }\end{array}$ & $0.517(0.006)$ & $0.583(<0.001)$ & $0.467(0.001)$ \\
\hline
\end{tabular}

ADC, apparent diffusion coefficient; OFC, orbitofrontal cortex; HbAlc, glycosylated hemoglobin.

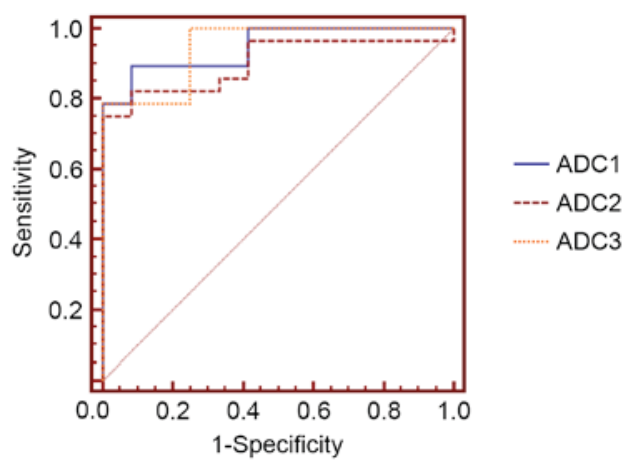

Figure 2. Receiver operating characteristic curves of ADC in regions of interest of the orbitofrontal cortex, cingulate gyrus and visual cortex in patients with type 2 diabetes with retinopathy. ADC1, ADC in the orbitofrontal cortex; $\mathrm{ADC} 2, \mathrm{ADC}$ in the cingulate gyrus; $\mathrm{ADC} 3, \mathrm{ADC}$ in the visual cortex; ADC, apparent diffusion coefficients.

cortices and visual cortices were significantly different among PDR, NPDR, diabetic without retinopathy and control groups $(\mathrm{P}<0.001$; Table II). SNK method comparison demonstrated that mean ADC values in cingulate gyri, orbitofrontal cortices and visual cortices were significantly higher in the PDR group than NPDR group, and significantly higher in the NPDR group than diabetic without retinopathy or control groups $(\mathrm{P}<0.05)$. Mean ADC values in thalami, coronae radiatae, dorsolateral frontal cortices and dorsomedial frontal cortices did not significantly differ among the four groups $(\mathrm{P}>0.05)$.

Correlations of HbAlc levels and disease duration with mean $A D C$ values in functional areas of the brain. Spearman's rho correlation was used to analyze correlations of HbAlc levels and disease duration with mean ADC values in the cingulate gyri, orbitofrontal cortices and visual cortices in PDR, NPDR and diabetic without retinopathy groups. HbAlc levels were positively correlated with mean ADC values in cingulate gyri $(r=0.287 ; \mathrm{P}=0.047)$, orbitofrontal cortices $(\mathrm{r}=0.328 ; \mathrm{P}=0.021)$, and visual cortices $(r=0.361 ; P=0.015$; Table III). Disease duration was also positively correlated with mean ADC values in cingulate gyri $(\mathrm{r}=0.517 ; \mathrm{P}=0.006)$, orbitofrontal cortices $(\mathrm{r}=0.583 ; \mathrm{P}<0.001)$ and visual cortices $(\mathrm{r}=0.467 ; \mathrm{P}=0.001)$. 
of ADC values was used to judge injuries to visual centers of the brain in type 2 diabetic patients with retinopathy. In the cingulate gyrus, the area under the ROC curve was 0.902 [95\% confidence interval $(\mathrm{CI})=0.766-0.973]$, with a diagnostic cut-off value of 753.000 , a sensitivity of 0.816 and a specificity of 0.851 (Fig. 2). In the orbitofrontal cortex, the area under the ROC curve was 0.946 (95\% CI=0.826-0.993), with a diagnostic cut-off value of 749.600 , a sensitivity of 0.855 and a specificity of 0.907 . In the visual cortex, the area under the ROC curve was 0.952 (95\% CI=0.826-0.993), with a diagnostic cut-off value of 739.800 , a sensitivity of 0.862 and a specificity of 0.914 .

\section{Discussion}

DR is a leading cause of blindness and visual impairment in patients with diabetes and is correlated with functional brain injuries (13). DWI is able to effectively provide information on pathological changes in the brain, including information on the diffusion rate of water molecules in tissues, transport of intracellular and extracellular water molecules, and microscopic and geometric structures of tissues, thus it offers an important basis for early diagnosis of diabetic encephalopathy (14). DWI may be used to calculate ADC, a measure of the diffusion capacity of water molecules in tissues, which can assess the degree of microstructural injuries in human tissues.

In the present study, mean ADC values were significantly higher in specific brain regions of individuals in the PDR group compared to the NPDR group. Mean ADC values were also significantly higher in the same brain regions of the NPDR group than in the diabetic without retinopathy or control groups. This result suggests that injuries to functional areas of the brain are correlated with DR. The results of the present study correlate with previous reports of increased ADC values in functional brain areas, which may be correlated with gliosis or nerve cell death $(10,15)$. ADC values in visual cortices of PDR and NPDR groups may be higher than those in diabetic without retinopathy and control groups as DR may reduce stimulation of visual cortices and lead to fine structural changes. Similarly, previous studies have demonstrated that visual dysfunction may lead to structural changes in the occipital cortex of amblyopic patients (16).

HbAlc levels represent a patient's blood sugar level over the past 3 months and are an important indicator of DR (17). Effective control of blood sugar may reduce the incidence of DR. In the present study, HbAlc levels were positively correlated with mean ADC values in the cingulate gyri, orbitofrontal cortices and visual cortices. Disease duration was also positively correlated with mean ADC values in these areas of the brain. This may be because longer disease duration affords greater diffusion capacity of water molecules, which is caused by neuronal degeneration in functional areas of the brain (18).

In the present study, ROC curve analysis was used to determine $\mathrm{ADC}$ values to judge functional brain injuries. In the cingulate gyrus, orbitofrontal cortex and visual cortex, all areas under ROC curves were $>0.9$, with high sensitivities and specificities, indicating that ADC may be used to assess functional brain injuries caused by DR. In conclusion, the results from the present study demonstrate that retinopathy in individuals with type 2 diabetes is correlated with functional brain injuries. DWI is an effective tool to assess such injuries in early DR and therefore may be a powerful technique for the prevention and treatment of DR.

\section{References}

1. Sivaprasad S, Gupta B, Crosby-Nwaobi R and Evans J: Prevalence of diabetic retinopathy in various ethnic groups: A worldwide perspective. Sur Ophthalmol 57: 347-370, 2012.

2. Liu L, Wu X, Liu L, Geng J, Yuan Z, Shan Z and Chen L: Prevalence of diabetic retinopathy in mainland China: A meta-analysis. PLoS One 7: e45264. 2012.

3. Xu J, Xu L, Wang YX, You QS, Jonas JB and Wei WB: Ten-year cumulative incidence of diabetic retinopathy. The Beijing Eye Study 2001/2011. PLoS One 9: e111320, 2014.

4. Liu L and Chen L: Awareness of diabetic retinopathy is the key step for early prevention, diagnosis and treatment of this disease in China. Patient Educ Couns 94: 284-285, 2014.

5. Rangarajan K, Das CJ, Kumar A and Gupta AK: MRI in central nervous system infections: A simplified patterned approach. World J Radiol 6: 716-725, 2014.

6. Mars RB, Jbabdi S, Sallet J, O'Reilly JX, Croxson PL, Olivier E, Noonan MP, Bergmann C, Mitchell AS, Baxter MG, et al: Diffusion-weighted imaging tractography-based parcellation of the human parietal cortex and comparison with human and macaque resting-state functional connectivity. J Neurosci 31: 4087-4100, 2011.

7. Taouli B: Diffusion-weighted MR imaging for liver lesion characterization: A critical look. Radiology 262: 378-380, 2012.

8. Vargas HA, Akin O, Franiel T, Mazaheri Y,Zheng J, Moskowitz C, Udo K, Eastham J and Hricak H: Diffusion-weighted endorectal MR imaging at $3 \mathrm{~T}$ for prostate cancer: Tumor detection and assessment of aggressiveness. Radiology 259: 775-784, 2011.

9. Shakir A, Aksoy D, Mlynash M, Harris OA, Albers GW and Hirsch KG: Prognostic value of quantitative diffusion-weighted MRI in patients with traumatic brain injury. J Neuroimaging 26: 103-108, 2016.

10. Dogan M, Ozsoy E, Doganay S, Burulday V, Firat PG, Ozer A and Alkan A: Brain diffusion-weighted imaging in diabetic patients with retinopathy. Eur Rev Med Pharmacol Sci 16: 126-131, 2012.

11. Buysschaert M, Medina JL, Buysschaert B and Bergman M: Definitions (and current controversies) of diabetes and prediabetes. Curr Diabetes Rev 12: 8-13, 2016.

12. Sacks DB, Arnold M, Bakris GL, Bruns DE, Horvath AR, Kirkman MS, Lernmark A, Metzger BE and Nathan DM; National Academy of Clinical Biochemistry; Evidence-Based Laboratory Medicine Committee of the American Association for Clinical Chemistry: Guidelines and recommendations for laboratory analysis in the diagnosis and management of diabetes mellitus. Diabetes Care 34: e61-e99, 2011.

13. Hajar S, Al Hazmi A, Wasli M, Mousa A and Rabiu M: Prevalence and causes of blindness and diabetic retinopathy in Southern Saudi Arabia. Saudi Med J 36: 449-455, 2015.

14. Glaser NS, Marcin JP, Wootton-Gorges SL, Buonocore MH, Rewers A, Strain J, DiCarlo J, Neely EK, Barnes P and Kuppermann N: Correlation of clinical and biochemical findings with diabetic ketoacidosis-related cerebral edema in children using magnetic resonance diffusion-weighted imaging. J Pediatr 153: 541-546, 2008.

15. Rock JP, Scarpace L, Hearshen D, Gutierrez J, Fisher JL, Rosenblum $\mathrm{M}$ and Mikkelsen T: Associations among magnetic resonance spectroscopy, apparent diffusion coefficients, and image-guided histopathology with special attention to radiation necrosis. Neurosurgery 54: 1111-1119, 2004.

16. Löwel S and Engelmann R: Neuroanatomical and neurophysiological consequences of strabismus: Changes in the structural and functional organization of the primary visual cortex in cats with alternating fixation and strabismic amblyopia. Strabismus 10: 95-105, 2002.

17. Nakagami T, Takahashi K, Suto C, Oya J, Tanaka Y, Kurita M, Isago C, Hasegawa Y, Ito A and Uchigata Y: Diabetes diagnostic thresholds of the glycated hemoglobin A1c and fasting plasma glucose levels considering the 5-year incidence of retinopathy. Diabetes Res Clin Pract 124: 20-29, 2017.

18. Ishii A, Takemoto M, Iida S and Kanatsuka A: Abdominal induration caused by repeated same site insulin injections is related to the HbAlc level and diurnal fasting blood glucose variation in type 2 diabetics. J Japan Diab Soc 58: 94-99, 2015. 